\title{
Metal Nanostructures on Ceramic Surfaces for Energy Applications
}

\author{
Joysurya Basu, Sanjit Bhowmick, Jonathan P. Winterstein, N. Ravishankar ${ }^{*}$ and C. Barry Carter \\ Department of Chemical, Materials and Biomolecular Engineering, 191 Auditorium Road, \\ University of Connecticut, Storrs-06269, CT, USA \\ * Materials Research Centre, Indian Institute of Science, Bangalore-560012, India \& Department \\ of Materials Science and Engineering and The Center for Future Energy Systems, Rensselaer \\ Polytechnic Institute, Troy-12180, NY, USA
}

One of the major advantages of using nanostructured materials is that their properties are not the same as the bulk material due to high surface to volume ratio [1-2]. For many applications, it is necessary to place the nanostructures on other supports/substrates. The idea of site-specific placement of nanostructures on other nanostructure to form hybrid nanostructures is relatively new and allows for a higher density of nanostructures per volume. Since site-specific placement of nanostructures is an important step for producing such hybrids, several techniques based on lithography have been developed that are often limited by cost-effectiveness and resolution. Nanostructured metals or ceramics on nanostructures have already been observed in several systems, e.g., Au-catalyzed growth of nanowires [3] or the chemical attachment of CdSe quantum dots on $\mathrm{ZnO}$ nanorods for solar cell application [4]. For larger more controlled arrays, site-specific placement of $\mathrm{Au}$ and $\mathrm{Ag}$ nanoparticles on reconstructed ceramic surfaces by parallel electron-beaminduced deposition technique [5] and metallic nanostructured pattern formation on reconstructed ceramic surfaces by dewetting [6] have also been demonstrated. The present paper reports the development of a new technique to deposit nanoscale catalyst particles on oxide nanocube supports for energy and environmental applications. The deposition is being carried out in situ by exploiting the electric-field singularity [7] at the sharp edges of the nanocubes to produce site-specific deposition on the cube edges.

A new technique of low-temperature hydrothermal growth has been developed to produce oxide nanocubes of materials that have broad potential as supports for catalysts and as proton conductor respectively in energy applications (e.g., pure and doped- $\mathrm{CeO}_{2}$ and $\mathrm{BaCeO}_{3}$ :Fig. 1). The details of the hydrothermal growth process have been reported elsewhere [8]. The hydrothermally grown particles have been deposited onto a TEM-ready sapphire substrate by dip-coating and also onto an electron-transparent silicon nitride membrane (Fig. 2). Electron-beam-induced deposition of Au and Co catalyst particles on the surfaces of the nanocubes is now being explored (Fig. 3), as is the dewetting of a thin metal film (Fig. 4). In situ imaging of such processes is generally expected to be limited by drift. Though there are several ex situ imaging and analytical techniques available today, each of them have limitations and may introduce large errors while characterizing such small length scales. High-resolution imaging must be used to characterize such nanomaterials. In this technique, it can be difficult to characterize both low- $Z$ and high- $Z$ atoms. In addition, aberrations, incorrect defocus setting and image delocalization will complicate the interpretation.

The present techniques should allow a higher number density of catalyst particles to be deposited on the surfaces of the active edges of the nanocubes. The composite nanostructures also could be combined with the development of porous membranes for thermal reforming of high-energy density liquid fuels using catalysis to convert the environmentally toxic by-products. Success of a scaled up process would improve the functionality of the membrane and reduce the required size.

\section{References}

[1] U. Sahayam, M. G. Norton, J. Mater. Sci., 43 (2008) 5395. 
[2] R. Z. Valiev, J. Mater. Sci., 42 (2007) 1483.

[3] J. Basu, R. Divakar, J. Nowak, S. Hofmann, A. Colli, A. Franciosi, C. B. Carter, J. Appl. Phys., 104, (2008) 064302.

[4] K.S. Leschkies, R. Divakar, J. Basu, E. Enache-Pommer, J.E. Boerker, C.B. Carter, U.R. Kortshagen, D.J. Norris, E.S. Aydil, Nano Lett., 7, (2007) 1793.

[5] J. Basu, C. B. Carter, R. Divakar, V. B. Shenoy, N. Ravishankar, Appl. Phys. Lett., 93, (2008) 133104.

[6] J. Basu, C. B. Carter, R. Divakar, B. Mukherjee, N. Ravishankar, (2009) (to be submitted).

[7] N. Ravishankar, V. B. Shenoy, C. B. Carter, Adv. Mater., 16, (2004) 76.

[8] J. Basu, J. P. Winterstein, S. Bhowmick, C. B. Carter, (2009) (submitted).

[9] Authors would like to acknowledge the University of Connecticut for support. JPW acknowledges the support from the Air Force in the form of NDSEG Fellowship.

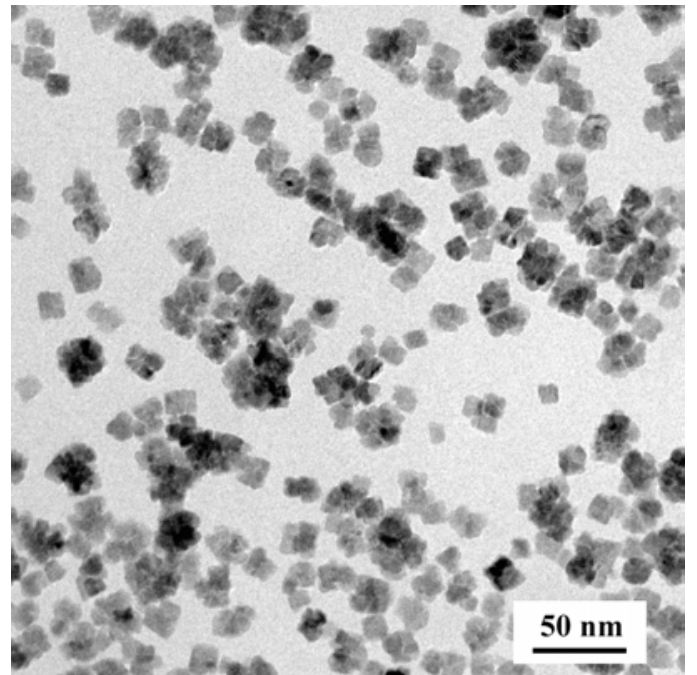

Figure 1: TEM micrograph of hydrothermally synthesized $\mathrm{BaCeO}_{3}$ nanocubes.

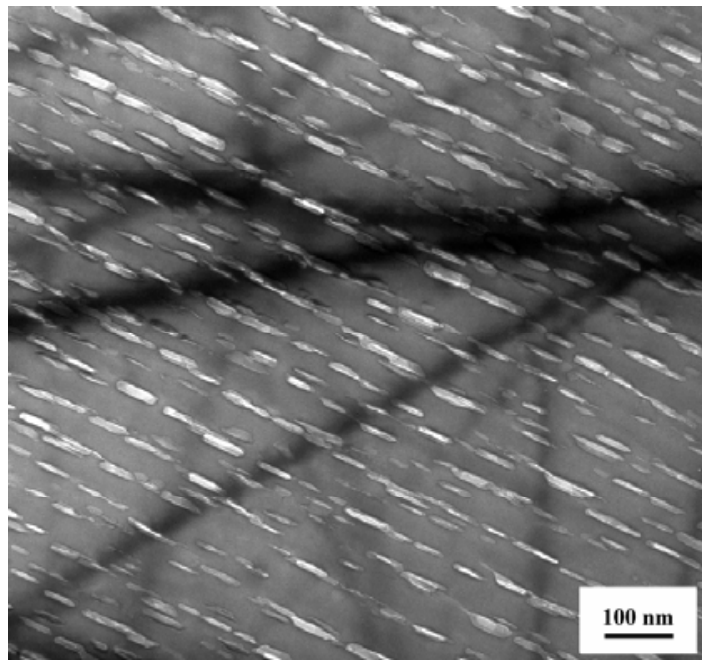

Figure 3: TEM image of Au nanoparticles deposited on a reconstructed $\mathrm{m}$-plane sapphire by electron beam.

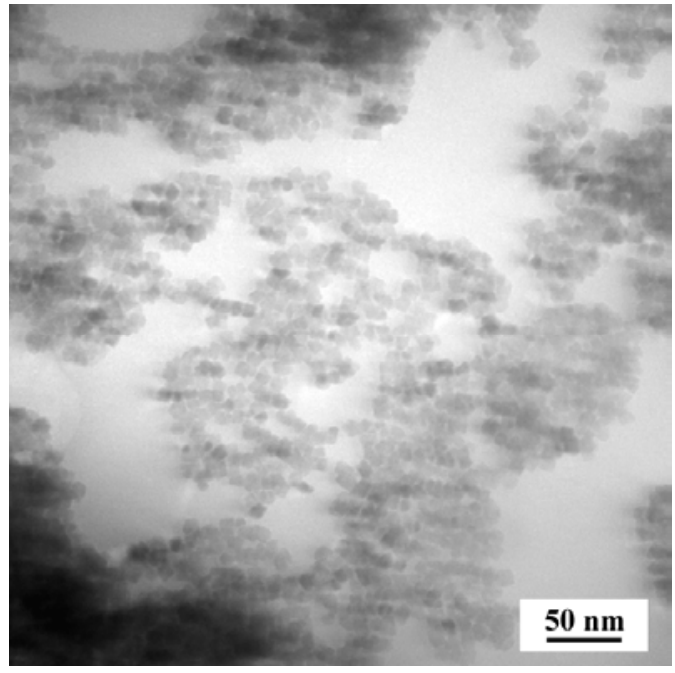

Figure 2: TEM micrograph of $\mathrm{CeO}_{2}$ nanocubes dip coated onto sapphire.

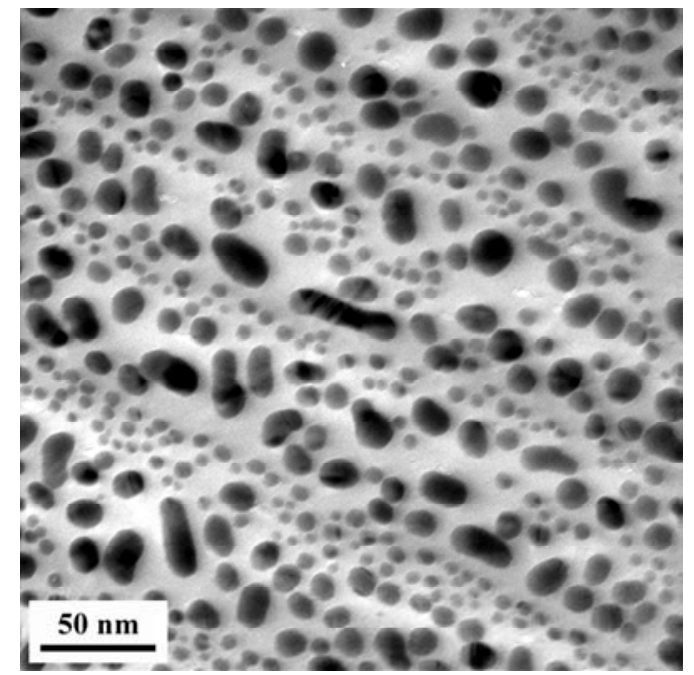

Figure 4: TEM micrograph of dewet Au nanoparticles on a reconstructed $\mathrm{m}$-plane sapphire. 